\title{
Efficacy of hydrophilic or lipophilic emulsions containing Echinacea purpurea extract in treatment of different types of pruritus
}

This article was published in the following Dove Press journal:

Clinical, Cosmetic and Investigational Dermatology

\author{
Ana Kilic \\ Anastasia Harder \\ Hubert Reich \\ Ulrich Knie \\ Clarissa Masur \\ Christoph Abels \\ Dr. August Wolff GmbH \& Co. KG \\ Arzneimittel, Bielefeld, Germany
}

Correspondence: Christoph Abels Dr. August Wolff GmbH \& Co. KG Arzneimittel, Sudbrackstr 56, Bielefeld 3361 I, Germany

Tel +4952I 8808437

Email christoph.abels@drwolffgroup.com
Background: Pruritus reduces quality of life and may occur at different sites of the body. To alleviate pruritus, lipid replenishing and rehydration of the skin is often unsatisfactory. Thus, products with additional antipruritic effects are needed.

Objectives: Antipruritic effects and cosmetic properties of two different emulsions, water-inoil (w/o) or oil-in-water (o/w), and a shampoo containing a lipophilic Echinacea purpurea root extract (Ec.-extract) were assessed in adults suffering from pruritus.

Methods: Adults $(\mathrm{n}=55)$ with pruritus of the body applied a w/o emulsion for 2 weeks. In a separate study, adults $(\mathrm{n}=33)$ with a pruritic scalp applied an o/w-emulsion for 4 weeks. In a third study, shampoo $(\mathrm{n}=34)$ was applied for 4 weeks. Objective (erythema, dryness, and papules) and subjective (intensity, duration, and burden of pruritus) parameters were assessed. Results: Treatment with the w/o emulsion significantly reduced erythema and dryness $(P<0.0001)$ as well as pruritus (in $93 \%$ of participants) on the body. Treatment with the o/wemulsion on the scalp significantly $(P<0.0001)$ reduced objective (erythema in $61 \%$ and dryness in $85 \%$ of participants) and subjective ( $85 \%$ of participants had reduced pruritus) parameters. Similar results in reduction of dryness ( $76 \%$ of participants) and pruritus ( $70 \%$ of participants) were seen after 4 weeks of shampoo use.

Conclusion: Independent from the type of emulsion (w/o or $\mathrm{o} / \mathrm{w}$ ), cosmetic products containing a proprietary Ec.-extract significantly reduced objective and subjective parameters in adults suffering from acute or chronic pruritus exhibiting excellent tolerability.

Keywords: acute pruritus, chronic pruritus, water-in-oil emulsion, oil-in-water emulsion, lipophilic Echinacea purpurea root extract

\section{Introduction}

Pruritus is a frequent and dominant symptom in many inflammatory skin diseases, for example, atopic eczema, psoriasis, ${ }^{1,2}$ while dry skin condition (xerosis cutis) can cause or aggravate pruritus. ${ }^{3}$ Pruritus can be localized or generalized, and it can occur as acute or chronic (when lasting more than 6 weeks). ${ }^{4,5}$ In addition, itch can be classified according to its origin as cutaneous (pruritoceptive), neuropathic, neurogenic, and psychogenic. ${ }^{4,6}$ Classically, histamine has been recognized as a mediator of itch, especially of acute pruritus. However, over the last years additional mediators (serotonin, substance $\mathrm{P}$, bradykinin, interleukin 13, and interleukin 31, toll like receptor 7) have been implicated in pruritus, nevertheless, the pathophysiology behind chronic pruritus is still not well understood. ${ }^{7-10}$ Due to the strong association of pruritus with dry skin, topical therapy using emollients or moisturizers is very often the treatment 
of choice to replenish lipids and rehydrate the stratum corneum. ${ }^{3,11}$ The aim for topical therapy of dry skin (xerosis cutis) or dry skin due to underlying skin diseases should thus be twofold: to repair the skin barrier and in addition to exhibit antipruritic effects, usually through addition of cosmetic or pharmacological active ingredients. ${ }^{3}$

Water-in-oil (w/o) emulsions are usually more effective than oil-in-water $(\mathrm{o} / \mathrm{w})$ emulsions since they replenish lipids to an impaired stratum corneum, which in turn reduces transepidermal water loss and thus w/o emulsions are a better choice for the treatment of very dry (xerotic) or atopic skin. ${ }^{12}$

As recently shown in a 3-month clinical trial, a w/o emulsion containing a proprietary Echinacea purpurea $(\mathrm{Ec})$ root extract (Ec.-extract) significantly reduced the local SCORAD and pruritus in patients with subacute or chronic atopic eczema (AE). ${ }^{13}$ Moreover, in contrast to a comparator product, improvements in AE symptoms lasted for up to 85 days (12 weeks) suggesting that beneficial effects of treatment with w/o emulsion containing Ec.-extract is beyond lipid replenishing and rehydration effects and could be due to anti-inflammatory effect of the extract. ${ }^{13}$

In order to assess whether a w/o emulsion containing Ec.-extract is safe and efficacious in treating other types of pruritus, not associated with AE, and affecting larger body surfaces, a study with adult patients suffering from either acute or chronic pruritus was performed.

In addition to pruritus of the body, a dry and itchy scalp is a very common complaint due to various causes (dermatologic, neuropathic, systemic, and psychogenic). ${ }^{14}$

Since a w/o emulsion is not cosmetically acceptable for scalp application, a novel o/w emulsion containing the Ec.extract was developed particularly for the treatment of a dry and itchy scalp, and its efficacy was tested in a second clinical study. Furthermore, we investigated the cosmetic acceptance and properties of a shampoo containing the Ec.-extract in participants with chronically itchy and a normal-to-dry scalp or scalp prone to eczema in a third study.

\section{Methods}

\section{Study to assess efficacy of the w/o emulsion containing Ec.-extract in adults suffering from pruritus Study design}

This open, nonrandomized, and noncontrolled study in adults suffering from acute pruritus (lasting less than 6 weeks) or chronic pruritus (lasting longer than 6 weeks) investigated a w/o emulsion with lipophilic Ec.-extract (Linola ${ }^{\circledR}$ Plus
Lotion, Dr August Wolff GmbH \& Co. KG Arzneimittel, Germany; INCI: aqua, decyl oleate, isopropyl myristate, carthamus tinctorius seed oil, hexyldecanol, hexyldecyl laurate, glycerin, polyglyceryl-3 polyricinoleate, sorbitan isostearate, citrus aurantium dulcis peel cera, zinc stearate, benzyl alcohol, magnesium sulfate, Ec.-extract, lecithin, tocopherol, ascorbyl palmitate, glyceryl oleate, glyceryl stearate, citric acid). The study protocol as well as other essential documents was approved by the Freiburg ethics commission international (feci code 017/1042) and was registered on clinicaltrials.gov registry with registration number NCT03477058.

Inclusion criteria were age $\geq 18$ years; sex $\sim 25 \%-50 \%$ male and $50 \%-75 \%$ female; complaining of unspecific pruritus (max. 50\% with acute pruritus [lasts less than 6 weeks] and at least $50 \%$ with chronic pruritus [lasts longer than 6 weeks]); phototype I-IV; in general good health and mental condition; personal informed consents of the subjects to participate in the study; personal presence on the predefined days at the institute; willing and capable to follow the study rules and a fixed schedule.

Of 55 participants included in the study, 18 (33\%) were male and 37 (67\%) female. Participants were between 20.2 and 78.6 years of age (average: $57.4 \pm 13.3$ years) with $95 \%$ of the participants suffering from chronic pruritus, while 5\% suffered from acute pruritus. Exclusion criteria included patients with AE, individuals using topical medication in the test area within 1 month, systemic medication with immunomodulators, and/or chemotherapeutic agents within 4 weeks and systemic medication with antibiotics or a change in the medication with anti-inflammatory agents within 2 weeks before starting the study, individuals with severe disorders within the last 12 months (cancer, dialysis patients) or with immunological disorders, pregnancy and breastfeeding, individuals participating in other studies on the body concurrently to this study or during the last 2 weeks before starting the study and individuals participating in a study with a pharmaceutical preparation within a period of at least 4 weeks prior to this study. Participants were selected in accordance with the recommendations of the Declaration of Helsinki and GCP guidelines and had to sign an informed consent before study entry.

Prior to the start of the study and during the study period, participants had to follow standardized instructions regarding skin care. At least 7 days beforehand and during the study period, participants had to refrain from changing their skin care and cleansing habits on body and face and from using peelings on the body. Twenty-four hours before the study, participants had to refrain from using any leave-on product 
on the body (excluding face). During the entire study, no other leave-on products than the test products were to be used, including oily skin cleansing products and peelings.

The product was applied at least once daily over a period of 2 weeks on the entire body and facultatively on the face. There were two assessment time points: at baseline (t0) and after 2 weeks of treatment (t1). Before assessments, participants acclimatized for at least 15 minutes at $21.5^{\circ} \mathrm{C}$ and $50 \%$ relative humidity and remained indoors until all assessments were completed.

A board-certified dermatologist evaluated the skin tolerance of the product regarding erythema, edema, dryness, papules, pustules, and fissures on both arms and legs at the start of the study and after 2 weeks of product application.

In addition, the dermatologist asked the participants to assess the sensory parameters of pruritus either burning or tension on arms and legs. At baseline, participants were requested to make their assessment based on the last 7 days before the start of the study. At the assessment after 2 weeks of product use, they were asked to consider the entire treatment period. Additionally, participants completed a questionnaire-based self-assessment covering intensity, duration, occurrence, and burden. Questionnaires can be found in supplement (Supplementary data S1 and S2).

Compliance was checked using the study diaries and weighing the product at the start and the end of the treatment period.

\section{Study to assess efficacy of the o/w emulsion containing Ec.-extract to combat scalp pruritus}

\section{Study design}

This open, nonrandomized, and noncontrolled study in adults suffering from dry, itchy scalp prone to AE or atopic dermatitis investigated a low fat $\mathrm{o} / \mathrm{w}$ emulsion containing a lipophilic Ec. extract (WO 4260; INCI: aqua, propanediol, isostearic acid, isostearyl alcohol, phenoxyethanol, Ec.- extract, carthamus tinctorius seed oil, isopropyl myristate, squalene, sodium polyacrylate, cetyl stearate, ethylhexyl cocoate, isostearyl isostearate, ethylhexylglycerin, cetyl alcohol, potassium cetyl phosphate, PPG-3 benzyl ether myristate, stearic acid, polysorbate 20). The study protocol as well as other essential documents was approved by the Freiburg ethics commission international (feci code 017/1035) and was registered on clinicaltrials.gov registry with registration number NCT03252730. Participants had to be users of hair tonic for dry scalp. Further inclusion criteria for the scalp studies were following: age $\geq 18$ years; sex: $\sim 50 \%$ male and $\sim 50 \%$ female; dry, itchy scalp prone to AE/atopic dermatitis; phototype I-IV; in general good health and mental condition; personal informed consents of the subjects to participate in the study; personal presence on the predefined days at the institute; willing and capable to follow the study rules and a fixed schedule. Of 33 participants included in this study, 16 (48\%) were male and 17 (52\%) were female. Participants were between 24.7 and 69.2 years old (on average $49.2 \pm$ 12.4 years).

Exclusion criteria were similar to those in the first study with following additions: users of sour hair rinse and other dermatological disorders on the scalp. Participants were selected in accordance with the recommendations of the Declaration of Helsinki and GCP guidelines and had to sign an informed consent before study entry.

The product was applied to the scalp once daily in the evening over a 4-week period. Prior to and during the course of the study, participants had to follow instructions regarding skin and hair care: at least 7 days before the start of the study and during the study they had to refrain from changing their shampoo and hair care products such as conditioner, serum, and hair masks. Participants had to refrain from washing their hair until the next morning. On the days of the visit, participants were requested to wash the hair at least 3 hours before the visit, at the same time for all visits. No hair styling products were to be used when visiting the study center.

Assessment time points were at baseline (t0) and after 2 (t1) and 4 (t2) weeks of treatment. Before each assessment, participants stayed in a climatized room with $21.5^{\circ} \mathrm{C}$ and $50 \%$ relative humidity for at least 15 minutes and they remained indoors until all assessments were completed. Compliance was checked using the study diaries and weighing the product at the start and the end of the treatment period.

A dermatologist assessed the tolerance of the product on the scalp: erythema, edema, dryness, scaliness, fissures, papules, and pustules on the scalp at the start of the study and after 2 and 4 weeks of product application. In addition, the dermatologist asked the participants to assess the sensory parameters pruritus, burning, and tension on the scalp. Same rating scale with 0.5 interval was used (for details see first study). At baseline, participants were requested to make their assessment based on the last 7 days before the start of the study and for the other two assessment time points based on the previous 2-week period.

Skin hydration was measured on the scalp (PinProbe, DermaLab $^{\circledast}$, ten single measurements) before starting the first product application and after 2 and 4 weeks of treatment. Further, after 4 weeks of treatment, participants completed 
a questionnaire-based self-assessment covering intensity of pruritus, burning, and tension on the scalp. Other questions to be answered related to the performance of the product. Questionnaire can be seen in supplementary material (Supplementary data S3).

The analysis of the study objectives was performed by SIT using the computer software.

\section{Study to assess tolerability and cosmetic properties of shampoo containing Ec.- \\ extract}

This open, nonrandomized, and noncontrolled in-use study investigating effect of shampoo containing Ec. extract on chronically itchy and normal-to-dry scalp was conducted on 34 subjects (18 female and 16 male subjects) (WO 5101, INCI aqua, mipa-laureth sulfate, peg-18 castor oil dioleate, propylene glycol, canola oil, sodium cocoamphoacetate, sodium chloride, triticum vulgare germ oil, peg-6 caprylic/ capric glycerides, glycerin, peg-55 propylene, glycol oleate, citric acid, disodium cocoyl glutamate, oleyl alcohol, potassium sorbate, polyquaternium-10, sodium cocoyl glutamate, limonene, Ec. root extract, sodium benzoate, parfum, linalool, citral, geraniol, citronellol, sodium acetate, isopropyl alcohol, coumarin, eugenol, tocopherol, hydrogenated palm glycerides citrate, farnesol). The study protocol as well as other essential documents were approved by the Freiburg ethics commission international (feci code 02018/1112). Only one subject did not finish the study completely and correctly, thus results of 33 subjects were included in the data analysis. These 33 subjects ( $52 \%$ female, $48 \%$ male) were between 20.2 and 70.2 years old (on average $48.9 \pm 14.2$ years). At least 14 days prior to the baseline assessment at $t 0$ and during the entire study period, coloration or dying of the hair was not allowed.

At least 7 days before the study, the subjects were refrained from changing their shampoo, hair care, and styling products such as conditioner, serum, or hair masks.

Inclusion and exclusion parameters were similar to those of other two studies. Assessment time points were at baseline (t0) and after 4 (t1) weeks of treatment (at least $3 \times$ a week). Before each assessment, participants stayed in a climatized room with $21.5^{\circ} \mathrm{C}$ and $50 \%$ relative humidity for at least 10 minutes, and they remained indoors until all assessments were completed. Dermatologist assessed appearance of the scalp at the $\mathrm{t} 0$ and $\mathrm{t} 1$ (erythema, edema, dryness, scaliness, papules, pustules, and fissures). In addition, the dermatologist asked the subject to assess pruritus, burning, and tension on the scalp. As in two previous studies, subjects had to assess the performance of the product in a questionnaire after 4-week treatment period (t1). Questionnaire can be seen in supplementary material (Supplementary data S4).

\section{Statistics}

For all studies, analysis of the study objectives was performed using the computer software Microsoft EXCEL ${ }^{\circledR}$ (version 2010) and STATISTICA ${ }^{\circledR}$ (version 13). Microsoft EXCEL was used for the calculation of the difference data and the descriptive statistics. STATISTICA was used for analyzing the significance of differences between the assessment times (Wilcoxon test for paired samples for discrete data) and the significance of the statements (Wilcoxon test for paired samples, chi square test - observed vs expected frequencies) in study I, Kolmogorov-Smirnov-test was used for analyzing the distribution of the data in study II, and Wilcoxon test for paired samples for discrete data was used for analyzing the significance of differences between the assessment times in study III. In the case of a $P$-value $<0.05$, a difference was accepted as statistically significant.

\section{Results \\ W/o emulsion containing lipophilic Ec.- extract significantly reduces pruritus, erythema, and skin dryness}

Two weeks after regular product use, the dermatological assessment revealed a statistically significant decrease in erythema and skin dryness $(P<0.0001)$ on arms and legs: 18 participants (33\%) had reduced erythema and 42 participants (76\%) had an improved assessment of skin dryness $>$ than -1 point (Table 1).

The results of the interviews conducted by the dermatologist regarding the participant's skin sensation showed that the 2-week treatment with a w/o emulsion containing Ec.-extract led to a statistically significant decrease in values for pruritus (the mean score for pruritus intensity decreased

Table I Frequency distribution of dermatologic grading of erythema and skin dryness after 2 weeks of regular use of waterin-oil emulsion containing Echinacea purpurea extract

\begin{tabular}{|l|l|l|}
\hline & Erythema & Dryness \\
\hline Frequency & No (\%) & No (\%) \\
\hline Improvement -3.0 to -4.0 & 0 & 0 \\
\hline Improvement -2.0 to -2.5 & $\mathrm{I}(2)$ & $\mathrm{I} 0(\mathrm{I} 8)$ \\
\hline Improvement $-\mathrm{I} .0$ to $-\mathrm{I} .5$ & $\mathrm{I}(3 \mathrm{I})$ & $32(58)$ \\
\hline No or very slight change $-0.5-0.5$ & $37(67)$ & $\mathrm{I} 3(24)$ \\
\hline Worsening I.0-I.5 & 0 & 0 \\
\hline Worsening 2.0-2.5 & 0 & 0 \\
\hline Worsening 3.0-4.5 & 0 & 0 \\
\hline
\end{tabular}

Notes: Erythema and dryness were determined in 55 adults suffering from chronic or acute pruritus. 
from 4.0 to $1.6[P<0.0001])$, burning $[P<0.0281]$, and tension $[P<0.0001])$. Pruritus was reduced in nearly all of the participants $(n=51 ; 93 \%)$, and the sensation of skin tension improved in 42 participants (76\%).

Subjective assessment based on a questionnaire-based selfassessment revealed a statistically significant reduction in pruritus intensity. At the beginning of the study, pruritus intensity ranged from slight to very strong, with 53\% reporting a clearly perceptible pruritus and $24 \%$ a strong or very strong pruritus. The 2-week application of the w/o emulsion containing Ec. extract led to a clear change in the pruritus intensity: strong pruritus was down to $2 \%$ and clearly perceptible pruritus down to $15 \%$. The majority of participants had no perceptible pruritus $(62 \%)$ or very slightly perceptible pruritus (18\%) after using w/o emulsion containing Ec.-extract for 2 weeks (Figure 1).

Overall, the questionnaire-based self-assessment showed a reduction of pruritus for $93 \%$ of the participants, which is in good accordance with the results obtained from the interviews conducted by the dermatologist.

At the same time, pruritus burden was also reduced. At the start of the study, $98 \%$ of the participants reported a burden due to pruritus and nearly half (47\%) rated the burden as being strong or very strong. This proportion was down to $2 \%$ after the 2 -week treatment, while $87 \%$ of the participants found the

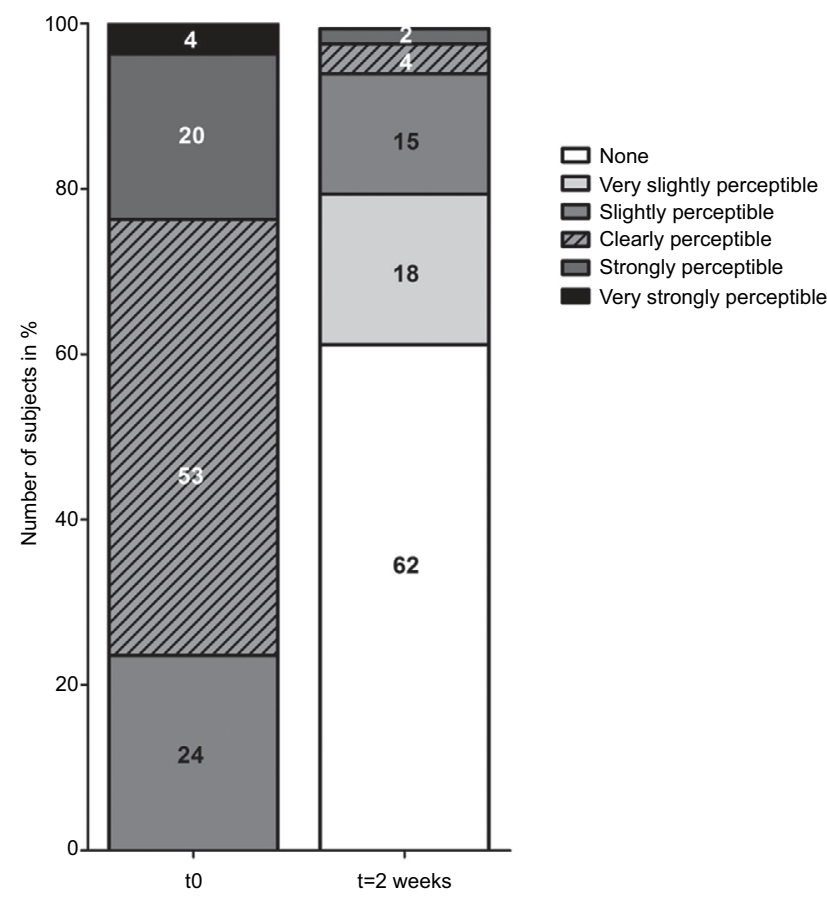

Figure I Percentage of adults $(n=55)$ suffering from chronic or acute pruritus who experience a given intensity of pruritus at baseline $(\mathrm{t} 0)$ and after 2 weeks of regular product use ( $\mathrm{t}=2$ weeks) (question PI Supplementary data $\mathrm{SI}$ ).

Notes: Intensity of pruritus was graded on a scale from I (none) to 6 (very strongly perceptible). pruritus no longer disturbing or even noted no burden at all. The mean score for pruritus burden decreased from 3.5 to 1.6 $(P<0.0001)$ on a scale from 1 (none) to 6 (unbearable). In total, $91 \%$ of the participants had a reduced pruritus burden.

Further, the duration of pruritus was shortened. At baseline the duration of pruritus was assessed as short term $(40 \%)$ or hourly $(49 \%)$ by most of the participants and constant pruritus was reported by $11 \%$. After 2 weeks of treatment, pruritus was not present in 34 out of $55(62 \%)$ of the participants. The remaining participants mostly assessed the duration of pruritus as only short term (35\%). Constant pruritus was no longer reported (Figure 2).

\section{Product performance of w/o emulsion containing E. purpurea extract is viewed favorably}

The participants were asked to rate the products performance in a questionnaire-based self-assessment. The majority of the participants $(69 \%)$ stated that the antipruritic effect of the product set in right after the first treatment and lasted for more than 24 hours.

Regarding the cosmetic properties, nearly two-third of the participants agreed that the emulsion spreads good or very good and that the emulsion absorbs fast or very fast.

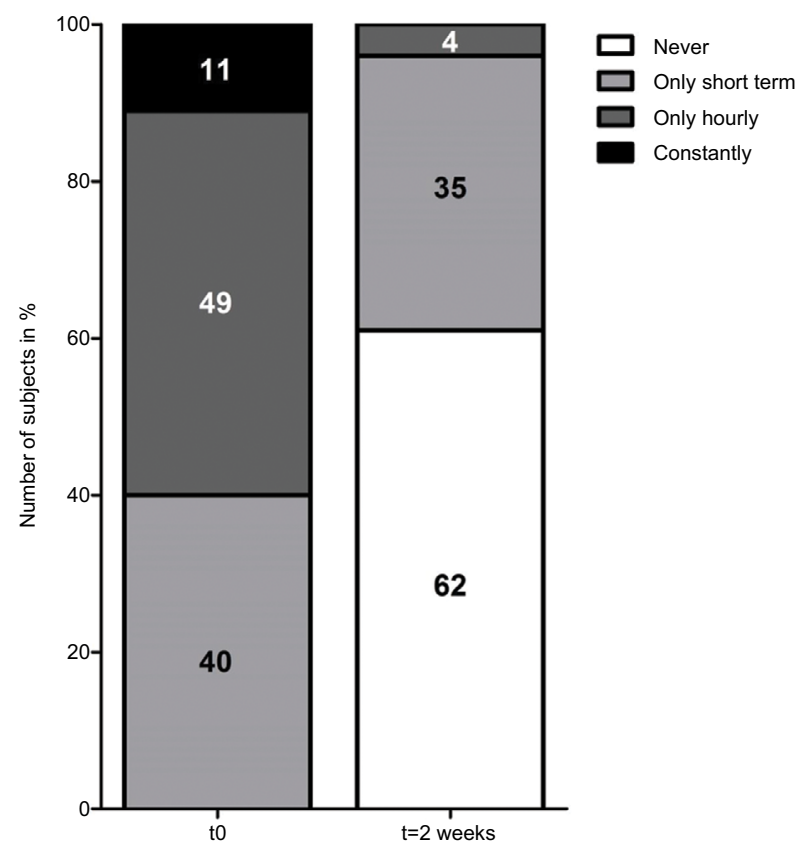

Figure 2 Percentage of adults $(n=55)$ suffering from chronic or acute pruritus who experience a given duration of pruritus at baseline $(\mathrm{t} 0)$ and after 2 weeks of regular product use ( $\mathrm{t}=2$ weeks) (question P2 Supplementary data $\mathrm{SI}$ ).

Notes: Intensity of pruritus was graded on a scale from I (never [pruritus no longer exists]) to 4 (constantly). 


\section{Scalp pruritus studies}

Low fat o/w emulsion containing Ec.-extract reduces erythema, dryness, and scaling on the scalp

The skin on the scalp was assessed by a dermatologist after 2 and 4 weeks of treatment. The results showed a statistically significant decrease for erythema $(P<0.0001)$, dryness $(P<0.0001)$, scaling $(P<0.0001)$, and papules $(P<0.0001)$ within the study period. Erythema was reduced in $45 \%$ of participants after 2 weeks and in $61 \%$ after 4 weeks, while dryness was reduced in $67 \%$ and $85 \%$ of participants after 2 and 4 weeks, respectively. At both time points, more than half of the participants noted an improvement in scaling (2 weeks: 57\%; 4 weeks: 61\%) (Figure 3A).

Papules were no longer observed after 2 weeks of treatment, corresponding to an improvement for $58 \%$ of the participants. There was one participant with pustules at baseline; these were no longer observed after the first 2-week treatment period.

In addition to the dermatological assessment, the skin hydration was measured. There was a statistically significant decrease in skin hydration within the first 2 weeks $(P<0.05)$ followed by a statistically significant increase in skin hydration $(P<0.05)$ between week 2 and week 4 (Figure 3B).

The analysis of the interviews regarding skin sensations conducted by the dermatologist revealed statistically significantly decreased values for the parameters pruritus, burning, and tension after 2 weeks and after 4 weeks of regular treatment. A total of $79 \%$ of participants had a reduction of pruritus after 2 weeks of treatment, increasing to $85 \%$ after a further 2 -week treatment period. The percentage of participants grading the reduction of the pruritus as moderate or high increased from $39 \%$ to $51 \%$ from week

A

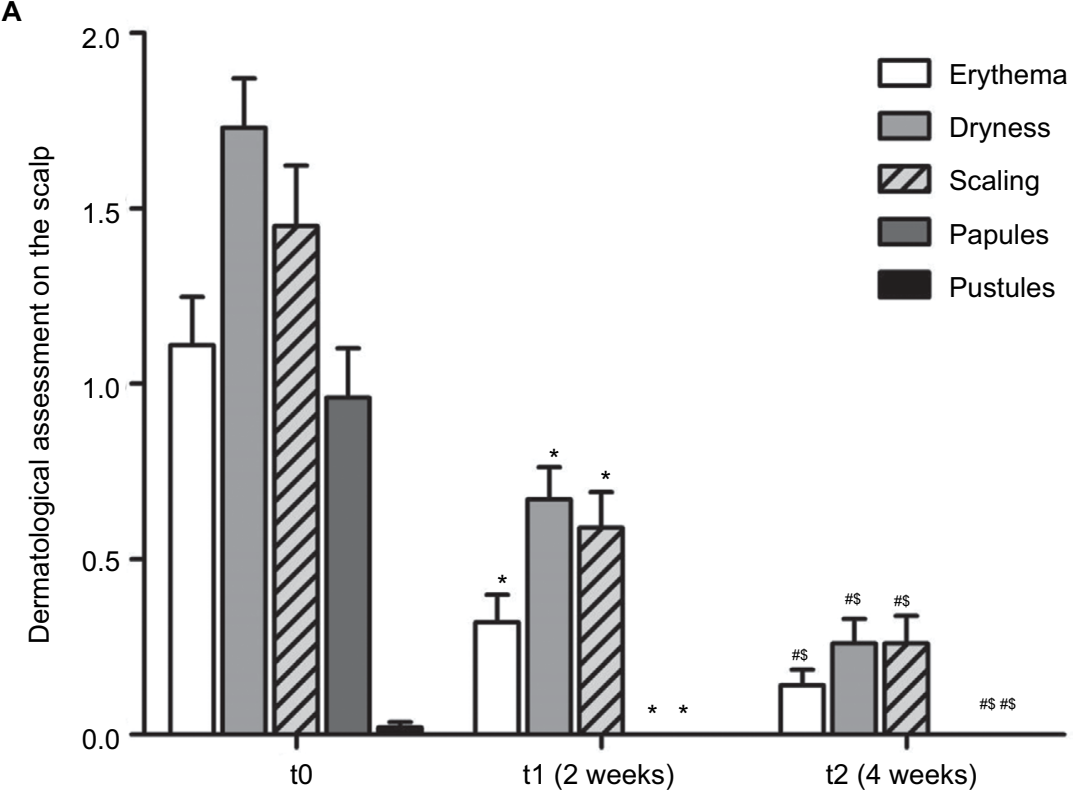

B

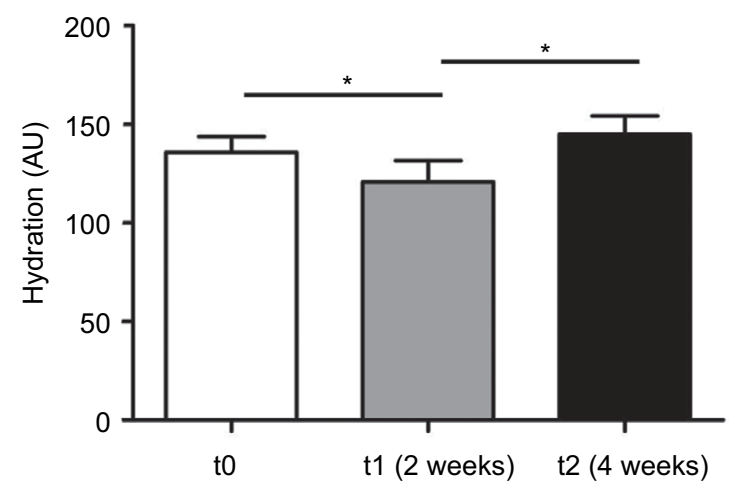

Figure 3 Dermatological assessment of erythema, dryness, scaling, papules, and pustules on the scalp at baseline (t0), 2 weeks ( $t$ l), and 4 weeks ( $t 2)$ after regular product use (A). Hydration of scalp at baseline ( $\mathrm{t} 0), 2$ weeks $(\mathrm{t} \mathrm{l})$, and 4 weeks $(\mathrm{t} 2)$ after regular product use (B).

Notes: Data are presented as a mean and SEM, $\mathrm{n}=33$ adults with pruritus of the scalp, ${ }^{*} P<0.05$ for $\mathrm{t} 1$ versus $\mathrm{t} 0,{ }^{\#} P<0.05$ for $\mathrm{t} 2$ versus $\mathrm{t} 0$ and ${ }^{\$} \mathrm{P}<0.05$ for $\mathrm{t} 2$ vs $\mathrm{t} \mathrm{l}$. 
2 to week 4 . The sensation of burning improved in $21 \%$ of the participants after 2 weeks of treatment and in $27 \%$ after 4 weeks of treatment. Every second participants also reported lessened skin tension ( $48 \%$ and $51 \%$ after 2 and 4 weeks, respectively).

Similar results were obtained from the subjective assessment via questionnaire-based interviews. The tonic was associated with positive effects on the participant's scalp condition: a vast majority reported reduced pruritus $(94 \%)$, burning (89\%), and tension (83\%). Skin dryness (88\%) and dandruff ( $83 \%)$ were also reduced. Overall, $88 \%$ were satisfied or very satisfied with the product's efficacy (Figure 4).

\section{Cosmetic properties of low fat o/w emulsion containing Ec.-extract are viewed favorably}

In general, the participants were very satisfied with the cosmetic performance of the product. Additionally, for most participants there was no negative impact on combing of the hair and they assessed the hair after washing to not differ significantly from "as usual".

\section{Shampoo containing Ec.-extract reduces dryness and scaling on the scalp}

The dermatological assessment of skin tolerance resulted in a statistically significant decrease in skin dryness $(P<0.0001)$ and scaliness $(P=0.0002)$ after 4 weeks of regular use of the test product. Regarding the parameters of erythema $(P=0.3271)$, edema $(P=0.6858)$, and papules $(P=1.0000)$ no significant changes for mean scores were observed after 4 weeks of regular product use. In $70 \%$ of participants pruritus

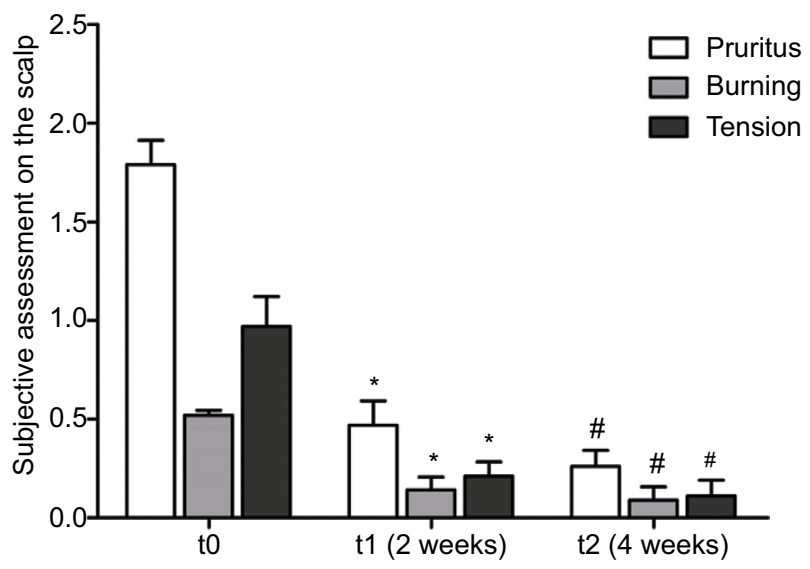

Figure 4 Subjective assessment of itching, burning, and tensions on the scalp at baseline (t0), 2 weeks ( $\mathrm{tl}$ ), and 4 weeks (t2) after regular product use.

Notes: Data are presented as a mean and SEM, $n=33$ adults with pruritus of the scalp, $* P<0.05$ for $\mathrm{tl}$ vs t $0, \# P<0.05$ for t 2 vs t 0 . lasted shorter, disappeared, or was distinctly lower. The same was seen for the symptoms burning and tension.

\section{Discussion}

Pruritus may be only a symptom but is a significant burden on quality of life caused by different skin diseases (eg, atopic dermatitis, xerosis cutis, psoriasis, or different systemic diseases, eg, liver disease, kidney failure). Once the cause of pruritus is known, a specific therapy can be induced. However, very often pruritus is chronic, and there is a need for effective medical skin care to reduce the burden of existing pruritus. Previous studies using a w/o emulsion containing a Ec.-extract significantly reduced AE symptoms including pruritus. ${ }^{13}$ Furthermore, the emulsion showed excellent tolerability. Thus, the aim was to test whether this Ec.-extract in different formulations may be used efficiently to treat larger body surfaces (and not only AE lesions), and whether it reliefs acute and chronic pruritus in adults not associated with AE.

Indeed, after application of the w/o emulsion containing E. purpurea root extract for 2 weeks, adults reported significant improvements regarding pruritus intensity and burden.

In agreement with the self-assessment by participants, interviews conducted by a dermatologist confirmed reduction of pruritus, burning, and tension. In addition, erythema and skin dryness were significantly reduced after using the Ec.extract containing w/o-emulsion for 2 weeks. Finally, patients were satisfied with the cosmetic properties of the product.

In the second, separate study, a newly developed low fat $\mathrm{o} / \mathrm{w}$ emulsion containing the Ec.-extract was tested on the dry and itchy scalp. Four week use of the product significantly decreased erythema, dryness, scaling, and papules on the scalp. Dandruff was also reduced and the majority of the participants also experienced a significant antipruritic effect of the emulsion. The percentage of participants benefiting from the regular use of the hair tonic was still increasing between week 2 and 4, which suggests beneficial long-term effects. The observed changes in skin hydration on the scalp (overall improvement with a reduction between baseline and the first assessment time-point at week 2) are difficult to interpret, as the study design did not include an untreated control. Considering the challenges of treating scalp pruritus ${ }^{15}$ the improvements documented here, with $79 \%$ of participants reporting an improvement after 2 weeks and $85 \%$ after 4 weeks of treatment, provide evidence that products containing this Ec.-extract are a promising additional treatment in the challenging setting of scalp pruritus because in case of hairy scalp, well-known barrier restoring effects of a w/o emulsion cannot be applied. This low-fat $\mathrm{o} / \mathrm{w}$ emulsion containing 
Ec.-extract used by patients suffering from scalp pruritus exhibited in addition to its efficacy good cosmetic properties according to the questionnaire. Similar results regarding reduction of dryness and pruritus of the scalp were also obtained by the use of the shampoo containing Ec.-extract. Future study using both products (shampoo and o/w emulsion) will show whether beneficial effect can be further improved.

Antipruritic effects exhibited by plant extracts are described in the literature. A cream containing the birch bark extract betulin (Imlan ${ }^{\circledR}$ Creme; Amryt AG, NiefernÖschelbronn, Germany) reduced pruritus in $52.7 \%$ of participants. ${ }^{16}$ The effects were strongly pronounced in participants who had pruritus due to dermatosis and participants with chronic scratch lesions. In the previous study, the used birch bark extract cream served as comparator product to the product containing Ec.-extract. Although both products reduced AE symptoms, effects seen with the birch bark extract containing cream diminished after 2 months, while the effects seen with Ec.-extract emulsion were persistent for up to 85 days, pointing to an anti-inflammatory and antipruritic effect of the Ec.-extract and not an emollient effect only. ${ }^{13}$

A split-scalp study on 30 individuals with itchy scalp investigated an aqueous leave-on tonic (EUCERIN ${ }^{\circledR}$-DermoCapillaire; Beiersdorf AG, Hamburg, Germany) containing multiple active ingredients including Glycyrrhiza inflata root extract (with licochalcone $\mathrm{A}$ as active ingredient). Treatment was performed only in a hair studio at the test center and trained study personnel applied the tonic to one side of the scalp and left the other side untreated. Under these controlled conditions participants reported reduction of scalp pruritus and reduction of scalp dryness. ${ }^{17}$ Moreover, initially postulated as treatment goal, there was no effect on the lipid content, but a clear reduction of free fatty acids was shown which could point to suboptimal barrier restoring effects. ${ }^{17} \mathrm{In}$ addition, a recent study investigating a emulsion containing pseudo-ceramide and eucalyptus extract showed effectively improved dry and scaly scalp as well as the quality of life of 34 participants with different skin conditions (atopic dermatitis, seborrheic dermatitis, pityriasis capitis). ${ }^{18}$

In contrast to the published study in atopic individuals, the objective and subjective effects seen in the two studies presented here (skin pruritus $>90 \%$ of participants; scalp pruritus $85 \%$ of participants) confirm that the Ec.-extract containing cosmetic products provide a treatment for both pruritic skin and itchy scalp independent from the vehicle (w/o or $\mathrm{o} / \mathrm{w}$ ). The beneficial clinical effects shown in the previous ${ }^{13}$ and current studies are further supported by recent findings that Ec.-extract inhibits mast cell degranulation. ${ }^{19}$
It might be speculated that Ec.-extract through its action on mast cells inhibits release of histamine, or other substance which subsequently might reduce pruritus..$^{20-22}$

In addition to Ec.-extract, both the w/o emulsion and low fat $\mathrm{o} / \mathrm{w}$ emulsion in this study contained linoleic acid, which may provide additional benefits for the regeneration of the skin barrier. Linoleic acid is well known to be essential for the epidermal permeability barrier, ${ }^{23,24}$ and it has been shown that oils with a higher linoleic acid to oleic acid ratio have better barrier repair potential. ${ }^{25}$ Furthermore, it was shown that the combination of linoleic acid and Ec.-extract is beneficial for barrier regeneration. The comparison to a preparation without Ec.-extract revealed that the positive effect is not purely emollient based, indicating synergistic effects of combining linoleic acid and Ec.-extracts in one product. ${ }^{13}$

Topically applied products to alleviate pruritus not only should provide an antipruritic effect but also have to meet customer expectations of medical skin care, in particular regarding the cosmetic properties. The tested w/o emulsion and low-fat $\mathrm{o} / \mathrm{w}$ emulsions met both needs by providing very good antipruritic effects and achieving a high user satisfaction regarding their cosmetic properties. Further, it is important to achieve a beneficial effect on a short time scale, as it is commonly felt that users must appreciate noticeable improvements within 14 days in order not to discontinue product use. ${ }^{26}$ The tested w/o emulsion achieved an effect already after first treatment in more than two-third (69\%) of the patients, and even $79 \%$ of patients reported significant reduction of pruritus within the first 2 weeks of using the hair tonic.

\section{Limitations}

Finally, we are aware that our study has limitations. Due to the fact that the investigated products are cosmetic products, the studies presented here did not include a control without Ec.extract and thus improvement of skin condition and reduction of pruritus seen in the studies may be due to effects of the entire cosmetic product. However, since the formulations where significantly different, w/o versus o/w versus shampoo, an effect primarily exhibited by the Ec.-extract at least for the o/w and shampoo is very likely, as shown recently for the w/o emulsion. ${ }^{13}$ All of our current studies covered maximally 4 weeks of use, so whether a longer-term use would result in additional or continued beneficial effects needs to be investigated.

\section{Conclusion}

The studies presented here investigated the use of medical skin care products containing a Ec.-extract in adults suffering from pruritus of different causes, but not related to $\mathrm{AD}$ of 
either body or scalp. Our results show that the application of either a w/o emulsion or a low-fat o/w emulsion containing Ec.-extract significantly reduced pruritus on body and scalp. In addition, the improvement was noticeable already after the first treatment and further improved within 14 days of product use. Based on the achieved antipruritic effect within 2 weeks and the good cosmetic properties, these medical skin care products show promise as adjuvant therapy to alleviate pruritus of body or scalp independent from underlying causes.

\section{Acknowledgments}

Medical writing support was provided by Doctor Alexander Boreham (co.medical, Berlin, Germany). Studies were funded by Dr. August Wolff GmbH \& Co. KG Arzneimittel, Germany.

\section{Disclosure}

$\mathrm{CA}$ and $\mathrm{UK}$ are named as inventors on the patent application; Soeberdt M, Abels C, Knie U, inventors. Patent WO 2017/037075A8. All of the authors are employees of Dr. August Wolff GmbH \& Co. KG Arzneimittel and report no other conflicts of interest in this work.

\section{References}

1. Greaves MW. Pathogenesis and treatment of pruritus. Curr Allergy Asthma Rep. 2010;10(4):236-242.

2. Savin JA. How should we define itching? J Am Acad Dermatol. 1998;39(2 Pt 1):268-269.

3. Metz M, Staubach P. Itch Management: Topical Agents. Curr Probl Dermatol. 2016;50:40-45.

4. Ständer S, Weisshaar E, Mettang T, et al. Clinical classification of itch: a position paper of the International Forum for the Study of Itch. Acta Derm Venereol. 2007;87(4):291-294.

5. Teofoli P, Procacci P, Maresca M, Lotti T. Itch and pain. Int J Dermatol. 1996;35(3):159-166.

6. Twycross R, Greaves MW, Handwerker H, et al. Itch: scratching more than the surface. QJM. 2003;96(1):7-26.

7. German Dermatological Society. S2k Leitlinie zur Diagnostik und Therapie des chronischen Pruritus. [S2k Guideline for the Diagnosis and Treatment of Chronic Pruritus]. Available from: https://www.awmf.org/leitlinien/ detail/11/013-048.html. Accessed October 17, 2018. German.

8. Yosipovitch G, Bernhard JD. Clinical practice. Chronic pruritus. NEngl J Med. 2013;368(17):1625-1634.

9. Kremer AE, Feramisco J, Reeh PW, Beuers U, Oude Elferink RP. Receptors, cells and circuits involved in pruritus of systemic disorders. Biochim Biophys Acta. 2014;1842(7):869-892.
10. Potenzieri C, Undem BJ. Basic mechanisms of itch. Clin Exp Allergy. 2012;42(1):8-19.

11. Grundmann S, Ständer S. Chronic pruritus: clinics and treatment. Ann Dermatol. 2011;23(1):1-11.

12. Lodén M, Maibach HI. Treatment of Dry Skin Syndrome. The Art and Science of Moisturizers. Heidelberg, Germany: Springer-Verlag; 2012.

13. Oláh A, Szabó-Papp J, Soeberdt M, et al. Echinacea purpurea-derived alkylamides exhibit potent anti-inflammatory effects and alleviate clinical symptoms of atopic eczema. J Dermatol Sci. 2017;88(1):67-77.

14. Bin Saif GA, Ericson ME, Yosipovitch G. The itchy scalp--scratching for an explanation. Exp Dermatol. 2011;20(12):959-968.

15. Beigi M, Weisshaar E. Pruritus of the scalp: simply pulling out your hair? Hautarzt. 2017;68(6):449-456.

16. Ständer S, Blome C, Phan NQ, et al. Antipruritische Effekte und individuelle Patientenbedürfnisse bei der topischen Therapie des chronischen Pruritus [Antipruritic Effects and Individual Needs of Patients on Topical Therapy for Chronic Pruritus]. Akt Dermatol. 2015;41:89-97.

17. Schweiger D, Baufeld C, Drescher P, et al. Efficacy of a new tonic containing urea, lactate, polidocanol, and glycyrrhiza inflata root extract in the treatment of a dry, itchy, and subclinically inflamed scalp. Skin Pharmacol Physiol. 2013;26(2):108-118.

18. Takagi $Y$, Ning $X$, Takahashi A, et al. The efficacy of a pseudo-ceramide and eucalyptus extract containing lotion on dry scalp skin. Clin Cosmet Investig Dermatol. 2018;11:141-148.

19. Gulledge TV, Collette NM, Mackey E, et al. Mast cell degranulation and calcium influx are inhibited by an Echinacea purpurea extract and the alkylamide dodeca-2E,4E-dienoic acid isobutylamide. J Ethnopharmacol. 2018;212:166-174.

20. Azimi E, Reddy VB, Pereira PJS, Talbot S, Woolf CJ, Lerner EA. Substance $P$ activates Mas-related $\mathrm{G}$ protein-coupled receptors to induce itch. J Allergy Clin Immunol. 2017;140(2):447-453.e3.

21. Gerber PA, Buhren BA, Cevikbas F, Bölke E, Steinhoff M, Homey B. Preliminary evidence for a role of mast cells in epidermal growth factor receptor inhibitor-induced pruritus. J Am Acad Dermatol. 2010;63(1):163-165.

22. Gupta K, Harvima IT. Mast cell-neural interactions contribute to pain and itch. Immunol Rev. 2018;282(1):168-187.

23. Darmstadt GL, Mao-Qiang M, Chi E, et al. Impact of topical oils on the skin barrier: possible implications for neonatal health in developing countries. Acta Paediatr. 2002;91(5):546-554.

24. Elias PM, Brown BE, Ziboh VA. The permeability barrier in essential fatty acid deficiency: evidence for a direct role for linoleic acid in barrier function. J Invest Dermatol. 1980;74(4):230-233.

25. Vaughn AR, Clark AK, Sivamani RK, Shi VY. Natural oils for skinbarrier repair: ancient compounds now backed by modern science. $\mathrm{Am}$ J Clin Dermatol. 2018;19(1):103-117.

26. Draelos ZD. An evaluation of topical 3\% salicylic acid and 1\% hydrocortisone in the maintenance of scalp pruritus. J Cosmet Dermatol. 2005; 4(3):193-197. 


\section{Supplementary materials Supplementary data SI}

Questionnaire for subjective assessment of pruritus:

(P1) Intensity: (1) none (pruritus no longer exists); (2) very slightly perceptible; (3) slightly perceptible; (4) clearly perceptible; (5) strongly perceptible; (6) very strongly perceptible

(P2) Duration: (1) never (pruritus no longer exists); (2) only short term; (3) only hourly; (4) constantly

(P3) Occurrence: (1) never (pruritus no longer exists); (2) only daytime; (3) only nighttime; (4) day and night; (5) only in cold conditions; (6) only in warm conditions; (7) in cold and warm conditions; (8) after showering; (9) further cause (to specify)

(P4) Burden: (1) none (pruritus no longer exists); (2) no longer disturbing; (3) does not bother me very much; (4) bothers me strongly; (5) bothers me very strongly; (6) unbearable

\section{Supplementary data S2}

Questionnaire for subjective assessment of product performance:

(Q1) How was the lotion to spread?

(1) very good; (2) good; (3) not too bad; (4) bad; (5) very bad

(Q2) How quickly was the lotion absorbed?

(1) very fast; (2) fast; (3) slow; (4) very slow

(Q3) How did your skin feel after applying the lotion?

(1) no longer dry; (2) less dry; (3) sufficiently greased; (4) too greasy

(Q4) How often did you use the lotion?

(1) once daily; (2) twice daily; (3) more than three times daily

(Q5) When did you usually use the lotion?

(1) only in the morning; (2) only in the evening; (3) in the morning and the evening; (4) without fixed rhythm

(Q6) How did you feel about the overall fragrance of the lotion?

(1) very good; (2) good; (3) not too bad; (4) unbearable

(Q7) How long did you benefit from the antipruritic effect of the lotion?

(1) only overnight; (2) only during daytime; (3) more than 24 hours (i.e., overnight and during daytime); (4) several hours (1-3 hours); (5) shortly (less than 1 hour); (6) not at all

(Q8) How quickly did the antipruritic effect of the lotion occur?
(1) right after first treatment; (2) only after several days of treatment; (3) not at all

(Q9) If you have already used a skin care product against pruritus, how do you assess its antipruritic effectiveness compared to the lotion?

(1) have not used an antipruritic skin care product; (2) the previous antipruritic skin care product is much less efficient;

(3) the previous antipruritic skin care product is less efficient;

(4) the previous antipruritic skin care product has the same effect; (5) the previous antipruritic skin care product is more efficient; (6) the previous antipruritic skin care product is much more efficient

(Q10) Would you further use the product if you had bought it?

(1) yes; (2) no

(Q11) Comments (open question)

\section{Supplementary data S3}

Questionnaire for subjective assessment of product performance:

(Q1) How do you assess the intensity of the Echinacea smell? (1) very good; (2) good; (3) not too bad; (4) too intense

(Q2) How do you assess the tonic's fragrance all together? (1) very good; (2) good; (3) not too bad; (4) unbearable

(Q3) How do you assess the tonic's spreadability?

(1) very good; (2) good; (3) not too bad; (4) bad; (5) very bad

(Q4) How easy was the tonic to be washed out with your shampoo?

(1) very good; (2) good; (3) not too bad; (4) bad; (5) not at all

(Q5) What was your hair like after you washed it with your shampoo?

(1) very much greasier than usual; (2) much greasier than usual;

(3) as usual; (4) drier than usual; (5) very much drier than usual

(Q6) How easy is your hair to comb?

(1) much better than usual; (2) better than usual; (3) as usual;

(4) worse than usual; (5) much worse than usual

(Q7) How fluffy was your hair?

(1) much better than usual; (2) better than usual; (3) as usual;

(4) worse than usual; (5) much worse than usual

(Q8) How much does the tonic affect your hair style?

(1) gives the hair style a much stronger hold; (2) gives the hair style a stronger hold; (3) hair style is not affected; (4) gives the hair style a weaker hold; (5) gives the hair style a much weaker hold 
(Q9) How was pruritus affected on your scalp?

(X) I don't suffer from pruritus on the scalp; (2) pruritus disappeared; (3) less pruritus; (4) no change; (5) increased pruritus; (6) pruritus is much worse

(Q10) How was burning affected on your scalp?

(X) I don't suffer from burning on the scalp; (2) burning disappeared; (3) less burning; (4) no change; (5) increased burning; (6) burning is much worse

(Q11) How was tension affected on your scalp?

(X) I don't suffer from tension on the scalp; (2) tension disappeared; (3) less tension; (4) no change; (5) increased tension; (6) tension is much worse

(Q12) How was dryness affected on your scalp?

(X) I don't suffer from dryness on the scalp; (2) dryness disappeared; (3) less dryness; (4) no change; (5) increased dryness; (6) dryness is much worse

(Q13) How was the formation of dandruff affected on your scalp?

(X) I don't suffer from dandruff on the scalp; (2) dandruff disappeared; (3) less dandruff; (4) no change; (5) increased dandruff; (6) formation of dandruff is much worse

(Q14) How happy were you overall with the tonic's efficacy?

(1) very satisfied; (2) satisfied; (3) not too bad; (4) not satisfied

(Q15) How long did you benefit from the tonic's effect?

(1) not at all; (2) only overnight; (3) only in the daytime;

(4) for 24 hours; (5) for several hours; (6) maximum one hour

(Q16) How often would you like to apply the tonic in the future?

(1) daily; (2) if required; (3) not at all

(Q17) Would you like to purchase the tonic if it was available in the market?

(1) yes; (2) no

\section{Supplementary data S4}

Questionnaire for subjective assessment of product performance:

(Q1) How satisfied are you with the combability of your hair?

(1) much better than before; (2) better than before, (3) equal as before; (4) worse than before; (5) much worse than before

(Q2) How did you feel the lightness of your hair?
(1) much better than before; (2) better than before; (3) equal as before; (4) worse than before; (5) much worse than before

(Q3) How did you feel the strength of the shampoo fragrance? (1) very good; (2) good; (3) not too bad; (4) too strong

(Q4) How did you feel about the overall fragrance of the Shampoo?

(1) very good; (2) good; (3) not too bad; (4) unbearable

(Q5) How did the pruritus of the scalp change?

(1) no pruritus before using the shampoo; pruritus after using the shampoo: (2) disappeared; (3) distinctly lower; (4) unchanged; (5) is stronger; (6) is much stronger

(Q6) How did the duration of the pruritus change on the scalp?

(1) no pruritus before using the shampoo; duration of the pruritus after using the shampoo: (2) much shorter; (3) shorter; (4) unchanged; (5) longer; (6) much longer

(Q7) How did the burning of the scalp change?

(1) no burning before using the shampoo; burning after using the shampoo: (2) disappeared; (3) distinctly lower; (4) unchanged; (5) stronger; (6) much stronger

(Q8) How did the duration of the burning change on the scalp?

(1) no burning before using the shampoo; duration of the burning after using the shampoo: (2) much shorter; (3) shorter; (4) unchanged; (5) longer; (6) much longer

(Q9) How did the tension of the scalp change?

(1) no tension before using the shampoo; tension after using the shampoo: (2) disappeared; (3) distinctly lower; (4) unchanged; (5) stronger; (6) much stronger

(Q10) How did the duration of the tension change on the scalp?

(1) no tension before using the shampoo; duration of the tension after using the shampoo: (2) much shorter; (3) shorter; (4) unchanged; (5) longer; (6) much longer

(Q11) Overall, how satisfied have you been with the effectiveness of the shampoo?

(1) very satisfied; (2) satisfied; (3) not too bad; (4) not at all (Q12) Would you like to buy the shampoo again if it is commercially available?

(1) yes; (2) no

(Q13) Comments, wishes (open question) 


\section{Publish your work in this journal}

Clinical, Cosmetic and Investigational Dermatology is an international, peer-reviewed, open access, online journal that focuses on the latest clinical and experimental research in all aspects of skin disease and cosmetic interventions. This journal is included on PubMed. The manuscript management system is completely online and includes a very quick and fair peer-review system, which is all easy to use. Visit http://www.dovepress.com/testimonials.php to read real quotes from published authors 